\title{
Changes of Th17/Treg ratio in the transition of chronic hepatitis B to liver cirrhosis and correlations with liver function and inflammation
}

\author{
HAIJUAN MOU $^{1 *}$, SONG WU ${ }^{2 *}$, GUANG ZHAO ${ }^{3}$ and JINDONG WANG ${ }^{4}$ \\ Departments of ${ }^{1}$ Public Health, ${ }^{2}$ Ultrasonic Medicine and ${ }^{3}$ Infectious Diseases, Yantai Infectious Disease Hospital, Yantai, \\ Shandong 264001; ${ }^{4}$ Department of General Surgery, Yantai Yeda Hospital, Yantai, Shandong 264006, P.R. China
}

Received January 3, 2018; Accepted January 7, 2019

DOI: $10.3892 /$ etm.2019.7299

\begin{abstract}
The changes in ratio of T helper 17 cells (Th17) to Treg cells in the transition of chronic hepatitis B (CHB) to liver cirrhosis, and the correlations with liver function and inflammation were investigated. A total of 20 normal subjects (control group), $35 \mathrm{CHB}$ patients (CHB group) and 40 post-hepatitis liver cirrhosis patients (liver cirrhosis group) were enrolled into this study. Liver function was measured through the levels of alanine aminotransferase (ALT) and aspartic transaminase (AST), and the hematoxylin and eosin (H\&E) staining method was used to detect the histopathological features. mRNA expression of inflammation-associated factors was detected using RT-PCR. The protein expression of nuclear factor $\kappa \mathrm{B}(\mathrm{NF}-\kappa \mathrm{B})$ was measured in liver tissues using the immunofluorescent method and western blot assay. In the CHB and liver cirrhosis groups, the increases in Th17 cells were more evident than those in Treg cells. Moreover, an evident increase in levels of ALT and AST was identified in the two groups. Structures of liver tissues in the CHB and liver cirrhosis groups were destroyed with damage to the cell nuclei. The expression of inflammation-associated factors were significantly elevated compared to those in the control group. $\mathrm{NF}-\kappa \mathrm{B}$ expressed in the $\mathrm{CHB}$ and liver cirrhosis groups was significantly higher than that in control group. The results of analysis of variance indicated that differences in the expression of interleukin (IL)-1 $\beta$, IL-6, tumor necrosis factor (TNF) $-\alpha$ and NF- $\kappa B$ in the three groups had statistical significance $(\mathrm{P}<0.01)$. In conclusion, transition from $\mathrm{CHB}$ to liver cirrhosis comes with significant changes in Th17/Treg
\end{abstract}

Correspondence to: Dr Jindong Wang, Department of General Surgery, Yantai Yeda Hospital, 11 Taishan Road, Yantai, Shandong 264006, P.R. China

E-mail: wangjindongyt@126.com

*Contributed equally

Key words: chronic hepatitis B, liver cirrhosis, Th17/Treg, liver function, inflammation ratio, which is correlated with a decrease in liver function, and also closely associated with the development and progression of inflammation.

\section{Introduction}

Chronic hepatitis B (CHB) is a common disease in clinical medicine. According to the World Health Organization, about 1 million people die each year from liver failure, liver cirrhosis and primary hepatocellular carcinoma caused by HBV infection, which seriously endanger human health $(1,2)$. Although some oral antiviral drugs and interferon have been widely used in clinic in recent years, the trend that CHB develops into cirrhosis, severe hepatitis has not been effectively curbed. Reducing the incidence of end-stage complications such as cirrhosis, effectively prolonging the life cycle of patients and improving the quality of life has become the key to be solved in the current medical field. Transition from CHB to liver cirrhosis involves many factors in key roles, such as changes in the ratio of T-helper 17 cells (Th17) to Treg cells, changes in the levels of alanine aminotransferase (ALT) and aspartic transaminase (AST) in liver function as well as the expression of various inflammation-associated factors (3-5).

In this study, with CHB patients and liver cirrhosis patients as subjects, we detected the changes in Th17/Treg ratio, variations in ALT and AST levels in liver function, and the expression of inflammation-associated factors [including interleukin (IL)-1 $\beta$, IL-6, tumor necrosis factor (TNF)- $\alpha$ and nuclear factor $\kappa \mathrm{B}(\mathrm{NF}-\kappa \mathrm{B})$ in liver functions] in liver tissues of all the groups to find the differences in expression during the transition from $\mathrm{CHB}$ to liver cirrhosis, and explore the correlations of Th17/Treg ratio with the liver function and inflammation in this process, aiming to provide new ideas and orientation for genetic diagnosis and treatment of $\mathrm{CHB}$ and liver cirrhosis.

\section{Patients and methods}

Sample collection

Patients. There were $35 \mathrm{CHB}$ patients and 40 post-hepatitis liver cirrhosis patients who were admitted to Yantai Infectious Disease Hospital (Yantai, China) between May 2010 and 
Table I. Primer sequences in RT-PCR.

Gene

Human GAPDH
Human $I L-1 \beta$
Human $I L-6$
Human $T N F-\alpha$

Forward primer (5'-3')

Reverse primer (5'-3')

RT-PCR, reverse transcription-polymerase chain reaction; GAPDH, glyceraldehyde-phosphate dehydrogenase; IL, interleukin; TNF, tumor necrosis factor.

July 2015, included in this study. Samples were collected from tissues resected during surgeries, fixed in $10 \%$ formaldehyde and embedded in paraffin. A total of 35 paraffin samples were collected from patients who underwent resection surgery in the hospital and were diagnosed as $\mathrm{CHB}$ through postoperative histopathological examination: There were 18 males and 17 females aged from 30 to 62 years. Additionally, 40 paraffin samples were collected from those who also underwent surgical resection in the hospital and were diagnosed as post-hepatitis liver cirrhosis: There were 27 males and 13 females aged from 36 to 69 years. In addition, 20 samples of normal liver tissues were collected from the liver transplantation center of the hospital as the control group, including 14 males and 6 females aged from 30 to 48 years.

The study was approved by the Ethics Committee of Yantai Infectious Disease Hospital and informed consents were signed by the patients or the guardians.

Major reagents. Roswell Park Memorial Institute (RPMI)-1640 medium and fetal bovine serum (FBS) were purchased from Gibco; Thermo Fisher Scientific, Inc. (Waltham, MA, USA); detection kits of AST and ALT were purchased from Nanjing Jiancheng Bioengineering Institute (Nanjing, China); bicinchoninic acid (BCA) detection kit of protein concentration and BeyoECL Plus kit were purchased from Beyotime Institute of Biotechnology (Haimen, China); extraction kit of tissue protein was purchased from Nanjing KeyGen Biotech Co., Ltd. (Nanjing, China); RNAiso Plus, PrimeScript ${ }^{\circledR}$ RT reagent kit with gDNA Eraser, SYBR ${ }^{\circledR}$ Premix Ex Taq $^{\mathrm{TM}}$ II (Tli RNaseH Plus) was purchased from Takara Biotechnology Co., Ltd. (Dalian, China); primary, secondary and fluorescent secondary antibodies of glyceraldehyde-phosphate dehydrogenase (GAPDH) and NF- $\kappa \mathrm{B}$ were purchased from Santa Cruz Biotechnology, Inc. (Dallas, TX, USA).

\section{Experimental methods}

Detection of Th17/Treg cell frequency in peripheral blood. Peripheral venous blood $(5 \mathrm{ml})$ was extracted from patients of the control, $\mathrm{CHB}$ and liver cirrhosis groups for isolating the peripheral blood mononuclear cells (PBMCs) in density of $2 \times 10^{6} / \mathrm{ml}$ through Ficoll density gradient centrifugation. The results were analyzed to identify the difference in frequencies of Th17 and Treg among the groups, and thus explore the correlations of Th17/Treg ratio with the liver function and inflammation.

Measurement of liver functions. According to the instructions of the kit, we assayed the enzymatic activity of AST and
ALT in serum to evaluate the damage to liver of patients in three groups.

Hematoxylin and eosin $(H \& E)$ staining. In all the groups, samples of liver tissues were embedded in paraffin to prepare the paraffin blocks, which were later sliced into $5 \mu \mathrm{m}$ sections as the blank sections. Thereafter, according to the regular method of histopathology, H\&E staining was performed for sections that were later placed under a microscope (x200, Olympus Corporation, Tokyo, Japan) for histopathological analysis.

Reverse transcription-polymerase chain reaction $(R T-P C R)$ analysis. Liver tissues in appropriate amount in the control, $\mathrm{CHB}$ and liver cirrhosis groups were transferred rapidly into the reagent supplemented with $1 \mathrm{ml}$ TRIzol to sufficiently grind the tissues into homogenate. Homogenate was placed at room temperature for $5 \mathrm{~min}$ RIPA lysis buffer, and then $12,000 \mathrm{x} g$ for $5 \mathrm{~min}$ at $4^{\circ} \mathrm{C}$. Supernatant was collected, mixed well with chloroform and placed at room temperature for $5 \mathrm{~min}$, followed by $12,000 \mathrm{x}$ f for $15 \mathrm{~min}$ at $4^{\circ} \mathrm{C}$. The same volume of isopropanol was added in the supernatant, and the solution was placed at room temperature for $10 \mathrm{~min}$, followed by $12,000 \mathrm{x} \mathrm{g}$ for $10 \mathrm{~min}$ at $4^{\circ} \mathrm{C}$. Sediment was collected, and mixed with $75 \%$ ethanol to rinse the sediment of RNA. Subsequently, RNase-free water was added to resolve the sediment, and the optical density (OD) was determined to calculate the $\mathrm{OD}_{260} / \mathrm{OD}_{280}$ ratio for measurement of RNA concentration. Under the manufacturer's instructions, stepwise amplification was performed for the primer sequences (Table I), and the product was collected for RT-PCR analysis.

Immunofluorescent assay. After being fixed in $10 \%$ formaldehyde for $48 \mathrm{~h}$, liver tissues in the three groups were embedded in paraffin regularly to prepare the sections $(5 \mu \mathrm{m})$. Paraffin sections, after being dewaxed in xylene and dehydrated in ethanol of gradient concentrations, underwent antigen retrieval, followed by rinsing with $0.01 \mathrm{M}$ phosphate-buffered saline (PBS; pH 7.4) three times (5 min/time). Thereafter, sections were blocked in a wet box containing $10 \%$ bovine serum albumin (BSA) at $37^{\circ} \mathrm{C}$ for $30 \mathrm{~min}$, followed by incubation with appropriately diluted (1:70) mouse anti-human GAPDH and NF- $\kappa$ B monoclonal antibodies (cat. nos. sc-365062 and sc-515045; Santa Cruz Biotechnology, Inc.) on a section at $4^{\circ} \mathrm{C}$ overnight. The section were rinsed with PBS ( $\mathrm{pH} 7.4)$ three times (5 min/time), fluorescent rabbit anti-mouse secondary polyclonal antibody (diluted at 1:100; cat. no. sc-2010; Santa Cruz Biotechnology, Inc.) were added on the sections in the dark for incubation in a wet box at $37^{\circ} \mathrm{C}$ for $2 \mathrm{~h}$. The sections were then rinsed using PBS ( $\mathrm{pH} 7.4)$ three times ( $5 \mathrm{~min} / \mathrm{time})$, 
Table II. Changes in Th17/Treg ratio in control group, CHB group and liver cirrhosis group.

\begin{tabular}{lccc}
\hline Index & Control group & CHB group & Liver cirrhosis group \\
\hline Thl7 $(\%)$ & $0.21 \pm 0.18$ & $0.22 \pm 0.21$ & $0.24 \pm 0.19$ \\
Treg $(\%)$ & $6.02 \pm 1.12$ & $6.73 \pm 1.68$ & $6.94 \pm 2.13$ \\
Thl7/Treg ratio & $35.21 \pm 13.18$ & $65.22 \pm 34.32$ & $69.25 \pm 43.64$ \\
\hline
\end{tabular}

Th17, T helper 17 cells; CHB, chronic hepatitis B.
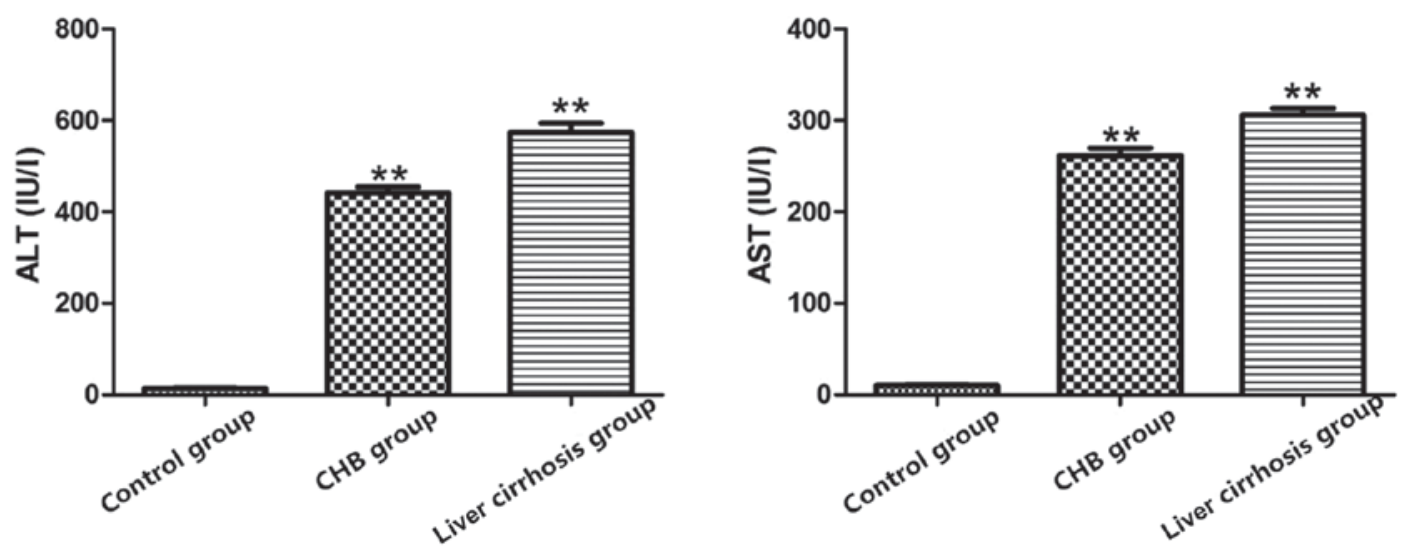

Figure 1. Detection of ALT and AST levels in control group, CHB group and liver cirrhosis group; ${ }^{* *} \mathrm{P}<0.01$ vs. control group. ALT, alanine aminotransferase; AST, aspartic transaminase; CHB, chronic hepatitis B.

4',6-diamidino-2-phenylindole (DAPI) was added in the dark for staining for $15 \mathrm{~min}$, and the sections were mounted with the $100 \%$ glycerol. Finally, with an upright fluorescent microscope (Olympus Corporation), the sections were observed and images were captured.

Western blot analysis. After being rinsed with ice-cold normal saline, samples of liver tissue in three groups were used for protein extraction in accordance with instructions of the extraction kit for total protein. Samples were ground sufficiently on ice with immunoprecipitation lysis containing phenylmethanesulfonyl fluoride (PMSF) and protease inhibitor. Tissues were then centrifuged at $12,000 \mathrm{x} \mathrm{g}$ for $10 \mathrm{~min}$ at $4^{\circ} \mathrm{C}$ for sufficient homogenization, and the supernatant was again centrifuged $12,000 \mathrm{x} \mathrm{g}$ at $4^{\circ} \mathrm{C}$ for $20 \mathrm{~min}$. After protein quantification with some of the supernatant, samples containing the same volume of total protein in the rest of supernatant were loaded in the wells for isolation through sodium dodecyl sulfate polyacrylamide gel electrophoresis (SDS-PAGE) under a constant voltage of $220 \mathrm{~V}$ until the bromophenol blue reached the bottom of the gel. According to molecular weight of the target proteins, gel was cut, and the proteins on the gel were transferred onto the polyvinylidene fluoride (PVDF) membrane. Then, PVDF membrane with protein was blocked in 5\% skimmed milk on a shaker at room temperature for $3 \mathrm{~h}$, and incubated with corresponding primary antibody $(1: 1,000)$ at $4^{\circ} \mathrm{C}$ overnight. Thereafter, membrane was rinsed with Tween-20 and Tris-buffer saline (TTBS) three times (10 $\mathrm{min} /$ time), and incubated with secondary antibody $(1: 2,000)$ for $1 \mathrm{~h}$ at room temperature. After the membrane was rinsed with TTBS three times (10 min/time), enhanced chemiluminescence (ECL) reagent was added for color development and photographing (ImageJ software; National Institutes of Health, Bethesda, MD, USA). The gray value of theband was scanned by Quantity One software (Bio-Rad Laboratories, Hercules, CA, USA).

Statistical analysis. Experiment data were presented as mean \pm standard deviation, and the results were analyzed with SPSS 17.0 software (SPSS, Inc., Chicago, IL, USA). A t-test was carried out for mean comparison between two groups, while one-way analysis of variance with LSD post hoc test for comparison of means of samples among groups, and p-test for pairwise comparison. $\mathrm{P}<0.05$ suggested that the difference had statistical significance.

\section{Results}

Results of changes in Th17/Treg ratio. As shown in Table II, frequency of Treg cells in the CHB and liver cirrhosis groups were significantly higher than that in the control group, and similar phenomena were also observed in a comparison of Th17 cell frequencies among these groups. In the CHB and liver cirrhosis groups, increases in Th17 cells were more evident than those in Treg cells, and the Th17/Treg ratio was also higher than that in control group.

Liver functions in the three groups. The results showed that compared with control group, increases in levels of AST and ALT in the CHB and liver cirrhosis groups were more evident $(\mathrm{P}<0.01)$, suggesting a decline in liver functions in $\mathrm{CHB}$ group and liver cirrhosis group, and severe damage to liver (Fig. 1). 

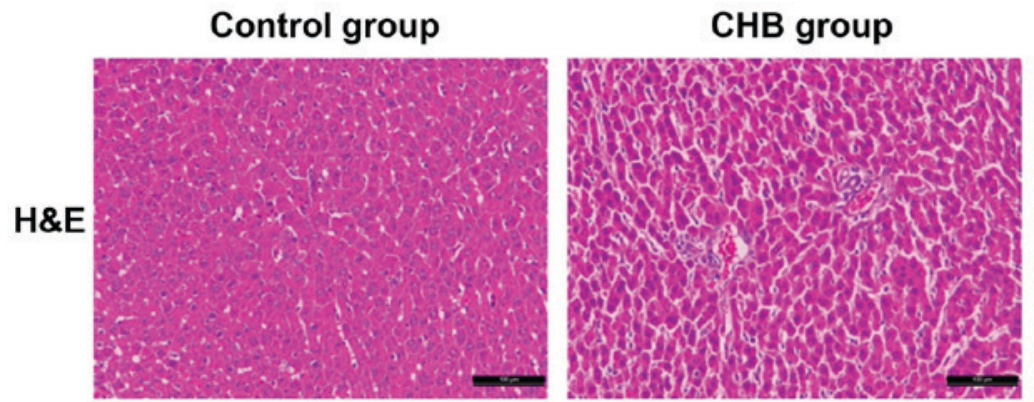

\section{Liver cirrhosis group}

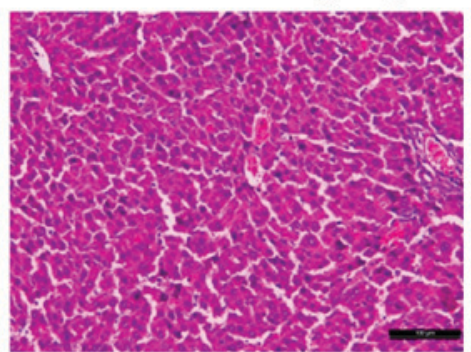

Figure 2. H\&E staining results of liver tissues in the control, CHB and liver cirrhosis groups (x200). H\&E, hematoxylin and eosin; CHB, chronic hepatitis B.
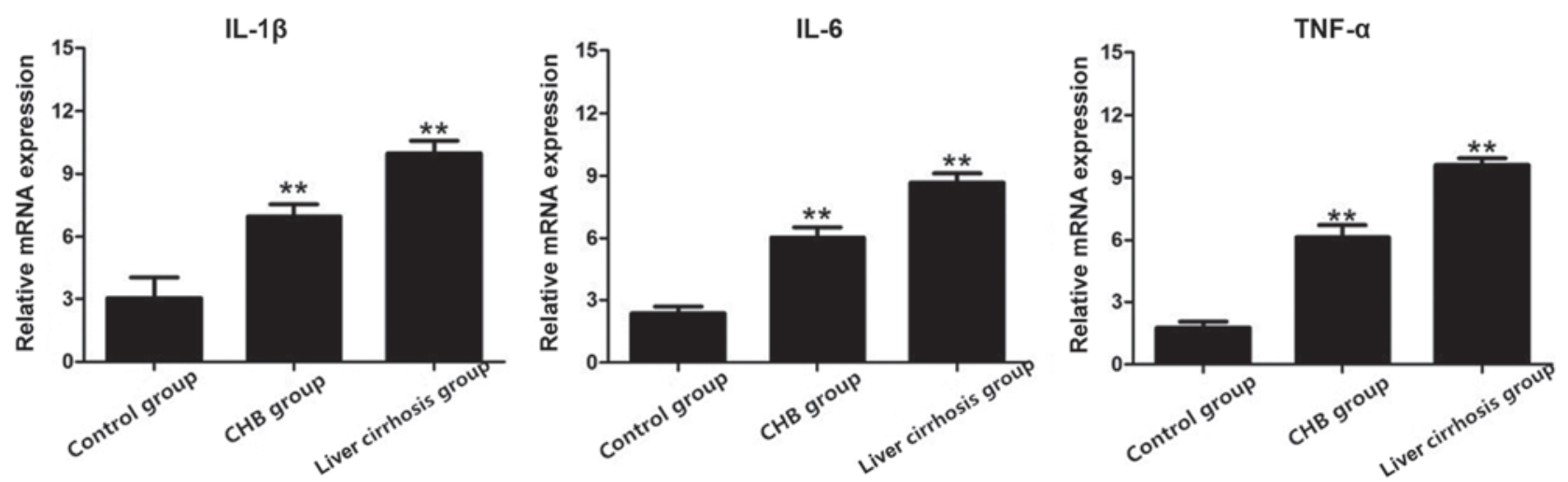

Figure 3. Expression of IL-1 $\beta$, IL-6 and TNF- $\alpha$ genes in the control, CHB and liver cirrhosis groups; ${ }^{* *} \mathrm{P}<0.01$ vs. control group. IL, interleukin; TNF, tumor necrosis factor; $\mathrm{CHB}$, chronic hepatitis B.
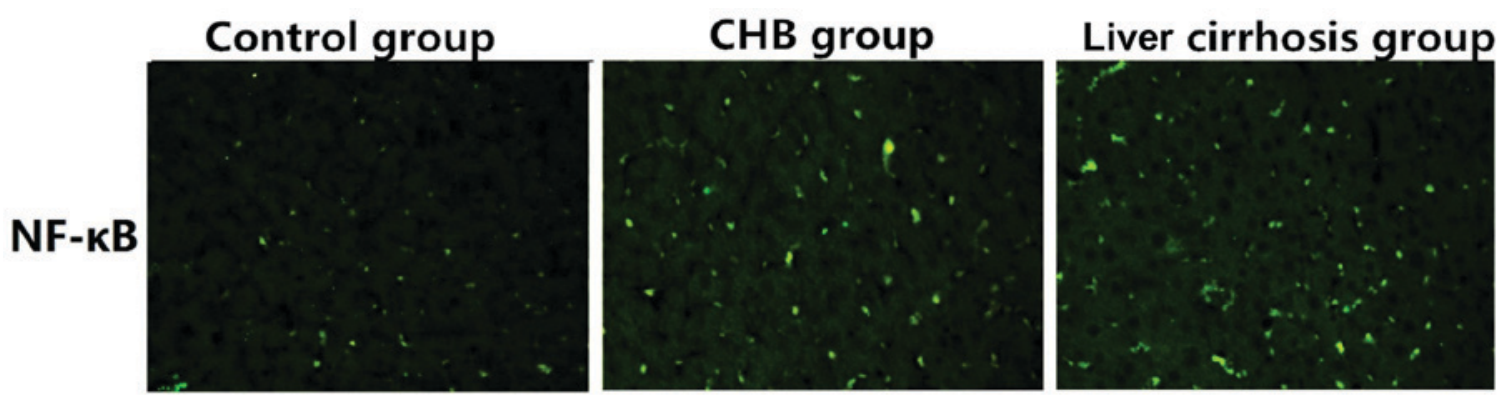

Figure 4. Expression of NF- $\mathrm{kB}$ in liver tissues of the control, CHB and liver cirrhosis groups (x200). NF- $\kappa \mathrm{B}$, nuclear factor $\kappa \mathrm{B}$; CHB, chronic hepatitis B.

Observation of pathological features through $H \& E$ staining. With the H\&E staining section in the control, CHB and liver cirrhosis groups, we aimed to identify the differences in pathological features of samples. Compared with the sections in control group, structures of liver tissues in sections of the $\mathrm{CHB}$ and liver cirrhosis groups were destroyed with hyperplasia of fibrous connective tissue, severe steatosis surrounding the central veins of liver, diversified size, hydropic degeneration and cloudy swelling of peripheral hepatocytes. In some massive necrosis area of hepatocytes, infiltration of inflammatory cells was also identified. As shown in Fig. 2, there were significant differences in the histopathological features of liver tissues among the three groups.

Results of RT-PCR. After RT-PCR, total RNA extracted from the three groups was used to detect the mRNA expression of IL-1 $\beta$, IL- 6 and TNF- $\alpha$, and the results showed that the mRNA expression of these indexes in the $\mathrm{CHB}$ and liver cirrhosis groups were obviously higher than those in the control group (Fig. 3).

Detection of the expression of $N F-\kappa B$ in control group, $\mathrm{CHB}$ group and liver cirrhosis group via immunofluorescence. As shown in Fig. 4, the expression of $\mathrm{NF}-\kappa \mathrm{B}$ in control group was significantly lower than those in the $\mathrm{CHB}$ and liver cirrhosis groupd, revealing that $\mathrm{NF}-\kappa \mathrm{B}$ is critical to the development and progression in $\mathrm{CHB}$, or even liver cirrhosis.

Protein expression of $\mathrm{NF}-\kappa \mathrm{B}$ in the control, $\mathrm{CHB}$ and liver cirrhosis groups. Results of the western blot analysis revealed the protein expression of $\mathrm{NF}-\kappa \mathrm{B}$ in the three groups. As shown in Fig. 5, the protein expression of $N F-\kappa B$ in liver tissues of the $\mathrm{CHB}$ and liver cirrhosis groups was far higher than that in control group. 


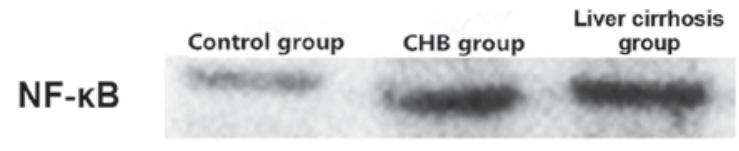

\section{GAPDH}

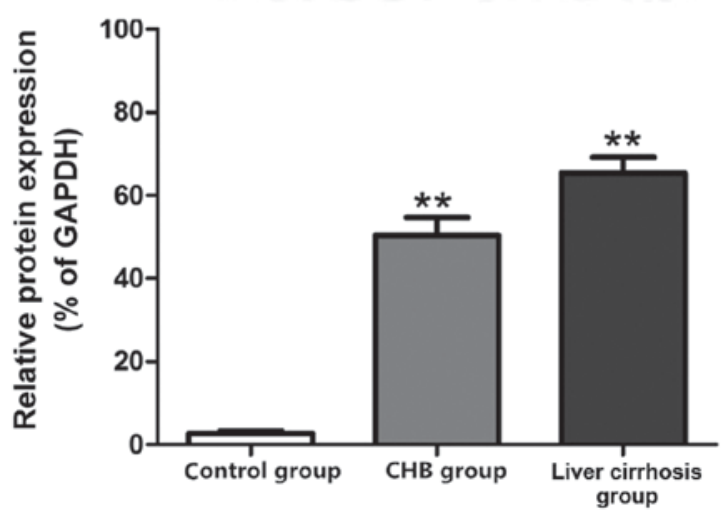

Figure 5. Protein expression of NF- $\mathrm{NB}$ in the control, $\mathrm{CHB}$ and liver cirrhosis groups; ${ }^{* *} \mathrm{P}<0.01$ vs. control group. $\mathrm{NF}-\kappa \mathrm{B}$, nuclear factor $\kappa \mathrm{B} ; \mathrm{CHB}$, chronic hepatitis B; GAPDH, glyceraldehyde-phosphate dehydrogenase.

\section{Discussion}

Liver is one of the major organs with strong defensive ability and regeneration ability in the body. Damage to liver affects the normal functions of body severely, and a variety of common liver diseases in clinic, including viral hepatitis, hepatic fibrosis, liver cirrhosis and liver cancer, seriously threaten the life and health of human beings (6-8). In the transition of CHB to liver cirrhosis, multiple factors are involved, including inflammation, oxidative stress, apoptosis and autophagy (9-11). Without effective treatment, it will evolve into liver cancer, leading to severe threat to life and health of human (12-14). Therefore, prophylaxis and treatment of liver diseases remain global problems, and it is imperative to develop an effective and safe treatment method for liver disease. Through years of studies, we have identified the genes that closely correlate with the disease, and appropriate intervention on these genes is expected to be a new orientation for treatment.

Inflammation is a complicated defensive response of living tissues with vascular system to destructive factors (15-17). Inflammatory factors mainly refer to the cytokines involved in inflammatory responses, and can be classified into several types: TNF- $\alpha$, IL-1 $\beta$, IL- 6 and TGF- $\beta$. The roles of inflammatory factors are mainly to induce the proliferation and differentiation of $\mathrm{T}$ lymphocytes in the body (18). Among various inflammation-associated cytokines, TNF- $\alpha$, IL- $1 \beta$ and IL- 6 are considered as the inflammatory factors with major effects $(19,20)$.

In this study, 20 healthy subjects (control group), $35 \mathrm{CHB}$ patients (CHB group) and 40 post-hepatitis liver cirrhosis patients (liver cirrhosis group) were enrolled into this study. In addition, liver functions were measured through the levels of ALT and AST with corresponding kits, and evident increases in levels of ALT and AST were identified in the CHB and liver cirrhosis groups in comparison with the levels in control group, suggestive of severe liver injury in the CHB and liver cirrhosis groups. Furthermore, H\&E staining results reflected directly the differences in histopathological features in the three groups, and RT-PCR analysis showed the significant differences in mRNA expression of inflammation-associated factors, including IL-1 $\beta$, IL- 6 and TNF- $\alpha$. Moreover, IL-1 $\beta$, IL- 6 and TNF- $\alpha$ were highly expressed in the CHB and liver cirrhosis groups, and expressed at low level in control group. The results of immunofluorescent assay and western blot assay showed that the expression of $\mathrm{NF}-\kappa \mathrm{B}$ in $\mathrm{CHB}$ group and liver cirrhosis group was significantly higher than that in control group.

In conclusion, transition from $\mathrm{CHB}$ to liver cirrhosis comes with significant changes in Th17/Treg ratio, which is correlated with the decline in liver function, and also closely associated with the development and progression of inflammation. In future, a new treatment method is expected to protect the liver functions through control of the deterioration of inflammation to protect the liver and reverse the liver disease.

\section{Acknowledgements}

Not applicable.

\section{Funding}

No funding was received.

\section{Availability of data and materials}

The datasets used and/or analyzed during the current study are available from the corresponding author on reasonable request.

\section{Authors' contributions}

HM wrote the manuscript. HM and SW performed PCR and western blot analysis. GZ and JW were responsible for immunofluorescent assay and H\&E staining analysis. All authors read and approved the final manuscript.

\section{Ethics approval and consent to participate}

The study was approved by the Ethics Committee of Yantai Infectious Disease Hospital (Yantai, China) and informed consents were signed by the patients or the guardians.

\section{Patient consent for publication}

Not applicable.

\section{Competing interests}

The authors declare that they have no competing interests.

\section{References}

1. Zou SS, Yang W, Yan HX, Yu LX, Li YQ, Wu FQ, Tang L, Lin Y, Guo LN, Zhou HB, et al: Role of $\beta$-catenin in regulating the balance between TNF- $\alpha$ - and Fas-induced acute liver injury. Cancer Lett 335: 160-167, 2013.

2. Yeh CH, Yang JJ, Yang ML, Li YC and Kuan YH: Rutin decreases lipopolysaccharide-induced acute lung injury via inhibition of oxidative stress and the MAPK-NF- $\kappa \mathrm{B}$ pathway. Free Radic Biol Med 69: 249-257, 2014. 
3. Jawan B, Kao YH, Goto S, Pan MC, Lin YC, Hsu LW, Nakano T, Lai CY, Sun CK and Cheng YF: Propofol pretreatment attenuates LPS-induced granulocyte-macrophage colony-stimulating factor production in cultured hepatocytes by suppressing MAPK/ERK activity and NF-kappaB translocation. Toxicol Appl Pharmacol 229: 362-373, 2008.

4. Mantena SK, King AL, Andringa KK, Eccleston HB and Bailey SM: Mitochondrial dysfunction and oxidative stress in the pathogenesis of alcohol- and obesity-induced fatty liver diseases. Free Radic Biol Med 44: 1259-1272, 2008.

5. Canbay A, Friedman S and Gores GJ: Apoptosis: The nexus of liver injury and fibrosis. Hepatology 39: 273-278, 2004.

6. Ferreira DM, Castro RE, Machado MV, Evangelista T, Silvestre A, Costa A, Coutinho J, Carepa F, Cortez-Pinto H and Rodrigues CM: Apoptosis and insulin resistance in liver and peripheral tissues of morbidly obese patients is associated with different stages of non-alcoholic fatty liver disease. Diabetologia 54: 1788-1798, 2011.

7. Malhi $\mathrm{H}$ and Gores GJ: Molecular mechanisms of lipotoxicity in nonalcoholic fatty liver disease. Semin Liver Dis 28: 360-369, 2008.

8. Tsuchida T, Shiraishi M, Ohta T, Sakai K and Ishii S: Ursodeoxycholic acid improves insulin sensitivity and hepatic steatosis by inducing the excretion of hepatic lipids in high-fat diet-fed KK-Ay mice. Metabolism 61: 944-953, 2012.

9. Rana M, Reddy SS, Maurya P, Singh V, Chaturvedi S, Kaur K, Agarwal H, Ahmad H, Naqvi A, Dwivedi AK, et al: Turmerone enriched standardized Curcuma longa extract alleviates LPS induced inflammation and cytokine production by regulating TLR4-IRAK1-ROS-MAPK-NFאB axis. J Funct Foods 16 $152-163,2015$.

10. Olleros ML, Vesin D, Fotio AL, Santiago-Raber ML, Tauzin S, Szymkowski DE and Garcia I: Soluble TNF, but not membrane TNF, is critical in LPS-induced hepatitis. J Hepatol 53: 1059-1068, 2010.

11. Gandhi A, Guo T, Shah P, Moorthy B and Ghose R: Chlorpromazine-induced hepatotoxicity during inflammation is mediated by TIRAP-dependent signaling pathway in mice. Toxicol Appl Pharmacol 266: 430-438, 2013.

12. Wang Y, Tu Q, Yan W, Xiao D, Zeng Z, Ouyang Y, Huang L, Cai J, Zeng X, Chen YJ and Liu A: CXC195 suppresses proliferation and inflammatory response in LPS-induced human hepatocellular carcinoma cells via regulating TLR4 MyD88-TAK1-mediated NF- $\kappa$ B and MAPK pathway. Biochem Biophys Res Commun 456: 373-379, 2015.
13. Shin WH, Jeon MT, Leem E, Won SY, Jeong KH, Park SJ, McLean C, Lee SJ, Jin BK, Jung UJ and Kim SR: Induction of microglial toll-like receptor 4 by prothrombin kringle-2: A potential pathogenic mechanism in Parkinson's disease. Sci Rep 5: 14764, 2015.

14. Gandoura S, Weiss E, Rautou PE, Fasseu M, Gustot T, Lemoine F, Hurtado-Nedelec M, Hego C, Vadrot N, Elkrief L, et al: Geneand exon-expression profiling reveals an extensive LPS-induced response in immune cells in patients with cirrhosis. J Hepatol 58: 936-948, 2013

15. Gu Q, Guan H, Shi Q, Zhang Y and Yang H: Curcumin attenuated acute Propionibacterium acnes-induced liver injury through inhibition of HMGB1 expression in mice. Int Immunopharmacol 24: 159-165, 2015.

16. Tao A, Song J, Lan T, Xu X, Kvietys P, Kao R, Martin C and Rui T: Cardiomyocyte-fibroblast interaction contributes to diabetic cardiomyopathy in mice: Role of HMGB1/TLR4/IL-33 axis. Biochim Biophys Acta 1852 (10 Pt A): 2075-2085, 2015.

17. Ramm S and Mally A: Role of drug-independent stress factors in liver injury associated with diclofenac intake. Toxicology 312 : 83-96, 2013.

18. Wang C, Song S, Zhang Y, Ge Y, Fang X, Huang T, Du J and Gao J: Inhibition of the Rho/Rho kinase pathway prevents lipopolysaccharide-induced hyperalgesia and the release of TNF- $\alpha$ and IL-1 $\beta$ in the mouse spinal cord. Sci Rep 5: 14553 , 2015.

19. Arrese M and Trauner M: Molecular aspects of bile formation and cholestasis. Trends Mol Med 9: 558-564, 2003.

20. Ma X, Wang J, He X, Zhao Y, Wang J, Zhang P, Zhu Y, Zhong L, Zheng Q and Xiao X: Large dosage of chishao in formulae for cholestatic hepatitis: A systematic review and meta-analysis. Evid Based Complement Alternat Med 2014: 328152, 2014.

This work is licensed under a Creative Commons Attribution-NonCommercial-NoDerivatives 4.0 International (CC BY-NC-ND 4.0) License. 